\title{
Computation of Betti Numbers of Monomial Ideals Associated with Cyclic Polytopes
}

\author{
N. Terai ${ }^{1}$ and T. Hibi ${ }^{2}$ \\ ${ }^{1}$ Department of Mathematics, Nagano National College of Technology, \\ Nagano 381, Japan \\ 2 Department of Mathematics, Hokkaido University, \\ Sapporo 060, Japan
}

\begin{abstract}
We give a combinatorial formula for the Betti numbers which appear in a minimal free resolution of the Stanley-Reisner ring $k[\Delta(\mathcal{P})]=A / I_{\Delta(\mathcal{P})}$ of the boundary complex $\Delta(\mathcal{P})$ of an odd-dimensional cyclic polytope $\mathcal{P}$ over a field $k$. A corollary to the formula is that the Betti number sequence of $k[\Delta(\mathcal{P})]$ is unimodal and does not depend on the base field $k$.
\end{abstract}

\section{Introduction}

Let $A=k\left[x_{1}, x_{2}, \ldots, x_{v}\right]$ denote the polynomial ring in $v$ variables over a field $k$, which will be considered to be the graded algebra $A=\bigoplus_{n \geq 0} A_{n}$ over $k$ with the standard grading, i.e., each $\operatorname{deg} x_{i}=1$. Let $\mathbf{Z}$ (resp. $\mathbf{Q}$ ) denote the set of integers (resp. rational numbers). We write $A(j), j \in \mathbf{Z}$, for the graded module $A(j)=\bigoplus_{n \in \mathbf{Z}}[\boldsymbol{A}(j)]_{n}$ over $\boldsymbol{A}$ with $[A(j)]_{n}:=A_{n+j}$. Let $I$ be an ideal of $A$ generated by homogeneous polynomials and let $R$ be the quotient algebra $A / I$. When $R$ is regarded as a graded module over $A$ with the quotient grading, it has a graded finite free resolution

$$
0 \rightarrow \bigoplus_{j \in \mathbf{Z}} A(-j)^{\beta_{h_{j}}} \stackrel{\varphi_{h}}{\longrightarrow} \cdots \stackrel{\varphi_{2}}{\longrightarrow} \bigoplus_{j \in \mathbf{Z}} A(-j)^{\beta_{1 j}} \stackrel{\varphi_{1}}{\longrightarrow} A \stackrel{\varphi_{0}}{\longrightarrow} R \rightarrow 0,
$$

where each $\bigoplus_{j \in \mathbf{Z}} A(-j)^{\beta_{i j}}, 1 \leq i \leq h$, is a graded free module of rank $0 \neq \sum_{j \in \mathbf{Z}} \beta_{i_{j}}<$ $\infty$, and where every $\varphi_{i}$ is degree-preserving. Moreover, there is a unique such resolution which minimizes each $\beta_{i}$; such a resolution is called minimal. If a finite free resolution (1) is minimal, then the homological dimension $\operatorname{hd}_{A}(R)$ of $R$ over $A$ is the nonnegative integer $h$ and $\beta_{i}=\beta_{i}^{A}(R):=\sum_{j \in \mathbf{Z}} \beta_{i_{j}}$ is called the $i$ th Betti number of $R$ over $A$. 
When $R$ is a Stanley-Reisner ring, i.e., $R=A / I$ where $I$ is generated by square-free monomials, its Betti numbers can be studied not only from an algebraic viewpoint but also from a topological and combinatorial one. It is an interesting problem to determine all the Betti numbers of the Stanley-Reisner rings for a good class of simplicial complexes.

In this paper, we give a combinatorial formula for the Betti numbers of the StanleyReisner ring of the boundary complex of an odd-dimensional cyclic polytope. Cyclic polytopes are important in combinatorics and have many good properties. For an evendimensional cyclic polytope, its associated Stanley-Reisner ring has a pure minimal free resolution. Thus its Betti numbers can be easily computed from its Hilbert function. See [Sc]. On the other hand, when a cyclic polytope has an odd dimension, its associated Stanley-Reisner ring does not have a pure minimal free resolution. We need much deeper analysis to calculate the Betti numbers.

\section{Simplicial Complexes and Hochster's Formula}

We first recall some notation on simplicial complexes and Hochster's topological formula on Betti numbers of Stanley-Reisner rings. We refer the reader to, e.g., [BHe], [H1], [Ho], and [St] for detailed information about combinatorial and algebraic background.

(1.1) A simplicial complex $\Delta$ on the vertex set $V=\left\{x_{1}, x_{2}, \ldots, x_{v}\right\}$ is a collection of subsets of $V$ such that

(i) $\left\{x_{i}\right\} \in \Delta$ for every $1 \leq i \leq v$ and

(ii) $\sigma \in \Delta, \tau \subset \sigma \Rightarrow \tau \in \Delta$.

Each element $\sigma$ of $\Delta$ is called a face of $\Delta$. Let \#( $\sigma)$ denote the cardinality of a finite set $\sigma$. We set $d=\max \{\#(\sigma) \mid \sigma \in \Delta\}$ and define the dimension of $\Delta$ to be $\operatorname{dim} \Delta=d-1$.

Given a subset $W$ of $V$, the restriction of $\Delta$ to $W$ is the subcomplex

$$
\Delta_{W}=\{\sigma \in \Delta \mid \sigma \subset W\}
$$

of $\Delta$. In particular, $\Delta_{V}=\Delta$ and $\Delta_{\varnothing}=\{\varnothing\}$.

Let $H_{i}(\Delta ; k)$ denote the $i$ th reduced simplicial homology group of $\Delta$ with the coefficient field $k$. Note that $\tilde{H}_{-1}(\Delta ; k)=0$ if $\Delta \neq\{\varnothing\}$ and

$$
\tilde{H}_{i}(\{\varnothing\} ; k)= \begin{cases}0 & (i \geq 0), \\ k & (i=-1)\end{cases}
$$

(1.2) Let $A=k\left[x_{1}, x_{2}, \ldots, x_{v}\right]$ be the polynomial ring in $v$ variables over a field $k$. Here, we identify each $x_{i} \in V$ with the indeterminate $x_{i}$ of $A$. Define $I_{\Delta}$ to be the ideal of $A$ which is generated by square-free monomials $x_{i_{1}} x_{i_{2}} \cdots x_{i_{r}}, 1 \leq i_{1}<i_{2}<\cdots<$ $i_{r} \leq v$, with $\left\{x_{i_{1}}, x_{i_{2}}, \ldots, x_{i_{r}}\right\} \notin \Delta$. We say that the quotient algebra $k[\Delta]:=A / I_{\Delta}$ is the Stanley-Reisner ring of $\Delta$ over $k$. In what follows we consider $A$ to be the graded algebra $A=\bigoplus_{n \geq 0} A_{n}$ with the standard grading, i.e., each $\operatorname{deg} x_{i}=1$, and may regard $k[\Delta]=\bigoplus_{n \geq 0}(k[\Delta])_{n}$ as a graded module over $A$ with the quotient grading. 
(1.3) Let $h=\operatorname{hd}_{A}(k[\Delta])$ denote the homological dimension of $k[\Delta]$ over $A$ and consider a graded minimal free resolution

$$
0 \longrightarrow \bigoplus_{j \in \mathbf{Z}} A(-j)^{\beta_{h_{j}}} \stackrel{\varphi_{h}}{\longrightarrow} \cdots \stackrel{\varphi_{2}}{\longrightarrow} \bigoplus_{j \in \mathbf{Z}} A(-j)^{\beta_{1_{j}}} \stackrel{\varphi_{1}}{\longrightarrow} A \stackrel{\varphi_{0}}{\longrightarrow} k[\Delta] \longrightarrow 0
$$

of $k[\Delta]$ over $A$. It is known that $v-d \leq h \leq v$.

We say that a simplicial complex $\Delta$ (or a Stanley-Reisner ring $k[\Delta]$ ) is CohenMacaulay (resp. Gorenstein) over a field $k$ if $h=v-d$ (resp. $h=v-d$ and $\beta_{v-d}=1$ ). Hochster's formula [Ho, Theorem (5.1)] guarantees that

$$
\beta_{i_{j}}=\sum_{W \subset V, \#(W)=j} \operatorname{dim}_{k} \tilde{H}_{j-i-1}\left(\Delta_{W} ; k\right) .
$$

Thus, in particular,

$$
\beta_{i}^{A}(k[\Delta])=\sum_{W \subset V} \operatorname{dim}_{k} \tilde{H}_{\#(W)-i-1}\left(\Delta_{W} ; k\right) .
$$

Some combinatorial and algebraic applications of Hochster's formula have been studied. See, e.g., [Ba], [BH1], [BH2], [?], [H2]-[H4], [TH1], and [TH2].

\section{Cyclic Polytopes}

In this section we briefly summarize the definition and basic facts of cyclic polytopes according to $[\mathrm{BL}]$ and $[\mathrm{Br}]$. See those references for detailed information.

(2.1) Let $\mathbf{R}$ denote the set of real numbers. For any subset $M$ of the $d$-dimensional Euclidean space $\mathbf{R}^{d}$, there is a smallest convex set containing $M$. We call this convex set the convex hull of $M$ and denote it by conv $M$. For $d \geq 2$ the moment curve in $\mathbf{R}^{d}$ is the curve parametrized by

$$
t \mapsto x(t):=\left(t, t^{2}, \ldots, t^{d}\right), \quad t \in \mathbf{R} .
$$

By a cyclic polytope $C(v, d)$, where $v \geq d+1$ and $d \geq 2$, we mean a polytope $\mathcal{P}$ of the form $\mathcal{P}=\operatorname{conv}\left\{x\left(t_{1}\right), \ldots, x\left(t_{v}\right)\right\}$, where $t_{1}, \ldots, t_{v}$ are distinct real numbers. It is well known that $C(v, d)$ is a simplicial $d$-polytope with the vertex set $\left\{x\left(t_{1}\right), \ldots, x\left(t_{v}\right)\right\}$, and its face lattice is independent of the particular values of $t$. Therefore its boundary complex is a simplicial complex and has the same combinatorial structure for any choices of vertices. We denote it by $\Delta(C(v, d))$.

Let $V=\left\{x_{1}, \ldots, x_{v}\right\}$ be the vertex set of $C(v, d)$. Let $W$ be a proper subset of $V$. A subset $X$ of $W$ of the form $X=\left\{x_{i}, x_{i+1}, \ldots, x_{j}\right\}$ is said to be a contiguous subset of $W$ if $i>1, j<v, x_{i-1} \notin W$, and $x_{j+1} \notin W$. The set $X$ is a left end-set of $W$ if $i=1$ and $x_{j+1} \notin W$, and a right end-set of $W$ if $j=v$ and $x_{i-1} \notin W$. We say that $X$ is a component of $W$ if $X$ is a contiguous subset or an end-set of $W$. A subset $X$ of $W$ is said to be even (resp. odd) if the number of elements in $X$ is even (resp. odd). The set $W$ can be written uniquely in the form $W=Y_{1} \cup X_{1} \cup \cdots \cup X_{n} \cup Y_{2}$, where $X_{i}, 1 \leq i \leq n$, 
is a contiguous subset of $W$, and $Y_{i}, i=1,2$, is an end-set of $W$ or an empty set. We quote two facts which are necessary later. We may abuse notation and call a subset $W$ of $V$ itself a face of $C(v, d)$ if conv $W$ is a face of $C(v, d)$.

Lemma 2.1 [Br, Theorem 13.7]. Let $W$ be an m-element subset of $V$, where $m \leq d$. Then $W$ is an $(m-1)$-face of $C(v, d)$ if and only if the number of odd contiguous subsets of $W$ is at most $d-m$.

Lemma 2.2 [ $\mathrm{Br}$, Corollary 13.8]. Let $m$ be an integer such that $1 \leq m \leq[d / 2]$. Then all m-element subsets of $V$ are $(m-1)$-faces of $C(v, d)$.

\section{Betti Numbers of Stanley-Reisner Rings Associated with Cyclic Polytopes}

In this section we compute the Betti numbers of a minimal free resolution of the StanleyReisner ring $k[\Delta(C(v, d))]$ of the boundary complex $\Delta(C(v, d))$ of the cyclic polytope $C(v, d)$.

We fix a field $k$. If the dimension $d$ is even, a minimal free resolution of $k[\Delta]$ is pure and the Betti numbers can be easily computed from the Hilbert function of $k[\Delta]$.

Proposition 3.1 [Sch]. Let $\Delta$ be the boundary complex $\Delta(C(v, d))$ of the cylic polytope $C(v, d)$, where $d \geq 2$ is even. Then a minimal free resolution of $k[\Delta]$ over $A$ is of the form

$$
\begin{aligned}
0 & \rightarrow A(-v) \rightarrow A\left(-v+\frac{d}{2}+1\right)^{\beta_{v-d-1}} \rightarrow \cdots \rightarrow A\left(-\frac{d}{2}-2\right)^{\beta_{2}} \rightarrow A\left(-\frac{d}{2}-1\right)^{\beta_{1}} \\
& \rightarrow A \rightarrow k[\Delta] \rightarrow 0
\end{aligned}
$$

where, for $1 \leq i \leq v-d-1$,

$$
\beta_{i}=\left(\begin{array}{c}
v-d / 2-1 \\
d / 2+i
\end{array}\right)\left(\begin{array}{c}
d / 2+i-1 \\
d / 2
\end{array}\right)+\left(\begin{array}{c}
v-d / 2-1 \\
i-1
\end{array}\right)\left(\begin{array}{c}
v-d / 2-i-1 \\
d / 2
\end{array}\right)
$$

Our formula on $\beta_{i}$ in Proposition 3.1 is, in fact, a little bit different from the one in [Sc]. However, it is easy to show that they are coincident.

If the dimension $d$ is odd, the minimal free resolution of $k[\Delta]$ is not pure, and the situation is much more complicated.

Now we state the main theorem in this paper.

Theorem 3.2. Let $\Delta$ be the boundary complex $\Delta(C(v, d))$ of the cyclic polytope $C(v, d)$, where $d \geq 3$ is odd. Then a minimal free resolution of $k[\Delta]$ over $A$ is of 
the form

$$
\begin{aligned}
0 & \rightarrow A(-v) \rightarrow A\left(-v+\left[\frac{d}{2}\right]+2\right)^{b_{v-d-1}} \oplus A\left(-v+\left[\frac{d}{2}\right]+1\right)^{b_{1}} \rightarrow \cdots \\
& \rightarrow A\left(-\left[\frac{d}{2}\right]-2\right)^{b_{2}} \oplus A\left(-\left[\frac{d}{2}\right]-3\right)^{b_{v-d-2}} \\
& \rightarrow A\left(-\left[\frac{d}{2}\right]-1\right)^{b_{1}} \oplus A\left(-\left[\frac{d}{2}\right]-2\right)^{b_{v-d-1}} \rightarrow A \rightarrow k[\Delta] \rightarrow 0,
\end{aligned}
$$

where, for $1 \leq i \leq v-d-1$,

$$
b_{i}=\left(\begin{array}{c}
v-[d / 2]-2 \\
{[d / 2]+i}
\end{array}\right)\left(\begin{array}{c}
{[d / 2]+i-1} \\
{[d / 2]}
\end{array}\right)
$$

Even if the geometric realization $|\Delta|$ of a simplicial complex $\Delta$ is a sphere, a Betti number of the Stanley-Reisner ring $k[\Delta]$ may depend on the base field $k$ in general. See Example 3.3 of [TH1]. However, as for the boundary complexes of cyclic polytopes we have the following result:

Corollary 3.3. Let $\Delta$ be the boundary complex $\Delta(C(v, d))$ of the cyclic polytope $C(v, d)$, where $d \geq 2$. Then all the Betti numbers of the Stanley-Reisner ring $k[\Delta]$ are independent of the base field $k$.

We prepare several lemmas to prove the theorem. We put $\Delta=\Delta(C(v, d))$ and $V=\{1,2, \ldots, v\}$ for simplicity, and fix an odd integer $d \geq 3$.

Lemma 3.4. If $v$ is odd and $W=\{1,3,5, \ldots, v\}$, then

$$
\tilde{H}_{[d / 2]-1}\left(\Delta_{W} ; k\right)=0 \text {. }
$$

Proof. We have $\tilde{H}_{[d / 2]-1}\left(\Delta_{W} ; k\right) \cong \tilde{H}_{[d / 2]}\left(\Delta_{V-W} ; k\right)$ by the Alexander duality theorem (see, e.g., p. 76 of [St]). Since $V-W=\{2,4, \ldots, v-1\}$, if $\sigma$ is a subset of $V-W$ with $\#(\sigma)>[d / 2]$, then $\sigma$ does not belong to $\Delta$ by Lemma 2.1. Thus we have $\tilde{H}_{[d / 2]}\left(\Delta_{V-W} ; k\right)=0$.

Lemma 3.5. If $v$ is even and $W=\{1,3,5, \ldots, v-1\}$, then

$$
\tilde{H}_{[d / 2]-1}\left(\Delta_{W} ; k\right)=0 \text {. }
$$

Proof. All the maximal faces of $\Delta_{W}$ are of the form $\{1\} \cup \sigma$, where $1 \notin \sigma$, $\sigma \subset W, \#(\sigma)=[d / 2]$. Thus $\Delta_{W}$ is a cone with apex $\{1\}$. Hence we have $\tilde{H}_{[d / 2]-1}\left(\Delta_{W} ; k\right)=0$.

Lemma 3.6. If $v$ is even and $W=\{2,4,6, \ldots, v\}$, then

$$
\tilde{H}_{[d / 2]-1}\left(\Delta_{W} ; k\right)=0 \text {. }
$$


Proof. As in Lemma 3.5, $\Delta_{W}$ is a cone with apex $\{v\}$. Hence we have $\tilde{H}_{[d / 2]-1}\left(\Delta_{W} ; k\right)$ $=0$.

Lemma 3.7. If $v$ is odd and $W=\{2,4,6, \ldots, v-1\}$, then

$$
\operatorname{dim}_{k} \tilde{H}_{[d / 2]-1}\left(\Delta_{W} ; k\right)=\left(\begin{array}{c}
{[v / 2]-1} \\
{[d / 2]}
\end{array}\right) .
$$

Proof. Let

$$
0 \rightarrow C_{d} \rightarrow C_{d-1} \rightarrow \cdots \rightarrow C_{1} \rightarrow C_{0} \rightarrow C_{-1} \rightarrow 0
$$

be the augmented chain complex of the simplicial complex $\Delta_{W}$ over $k$. Then we have $C_{[d / 2]}=0$ and, for $j<[d / 2]$, all the $(j+1)$-subsets of $W$ form a basis of $C_{j}$ as a vector space by Lemmas 2.1 and 2.2. Thus we have $\tilde{H}_{j}\left(\Delta_{W} ; k\right)=0$ for all $j<[d / 2]-1$. Hence, the Euler-Poincaré formula (see, e.g., p. 223 of [BHe]) gives

$$
\begin{aligned}
& \operatorname{dim}_{k} \tilde{H}_{[d / 2]-1}\left(\Delta_{W} ; k\right) \\
& =(-1)^{[d / 2]}\left(\left(\begin{array}{c}
{[v / 2]} \\
0
\end{array}\right)-\left(\begin{array}{c}
{[v / 2]} \\
1
\end{array}\right)+\left(\begin{array}{c}
{[v / 2]} \\
2
\end{array}\right)-\cdots+(-1)^{[d / 2]}\left(\begin{array}{c}
{[v / 2]} \\
{[d / 2]}
\end{array}\right)\right) \\
& =\left(\begin{array}{c}
{[v / 2]-1} \\
{[d / 2]}
\end{array}\right) .
\end{aligned}
$$

Lemma 3.8. Let $W$ be a nonempty proper subset of $V$ with a unique decomposition

$$
W=Y_{1} \cup X_{1} \cup X_{2} \cup \cdots \cup X_{n} \cup Y_{2}
$$

for some $n \geq 0$, where $X_{i}, 1 \leq i \leq n$, is a contiguous subset and $Y_{i}, i=1,2$, is an end-set or an empty set. Then

$$
\operatorname{dim}_{k} \tilde{H}_{[d / 2]-1}\left(\Delta_{W} ; k\right)= \begin{cases}\left(\begin{array}{l}
n-1 \\
{[d / 2]}
\end{array}\right) & \text { if } Y_{1}=\varnothing \text { and } Y_{2}=\varnothing \\
0 & \text { otherwise }\end{cases}
$$

where we define $\left(\begin{array}{l}n-1 \\ {[d / 2]}\end{array}\right)=0$ if $n-1<[d / 2]$.

Proof. We prove the lemma by induction on the number $v$ of vertices. First let $v=d+1$. Then $C(v, d)$ is a $d$-simplex. Thus $\tilde{H}_{[d / 2]-1}\left(\Delta_{W} ; k\right)=0$ for every subset $W \subset V$. Since

$$
n \leq\left[\frac{v-1}{2}\right]=\left[\frac{d}{2}\right]
$$

the lemma holds.

Next let $v>d+1$. Let

$$
V-W=X_{1}^{\prime} \cup X_{2}^{\prime} \cup \cdots \cup X_{n+1}^{\prime}
$$


be a unique decomposition, where $X_{i}^{\prime}, 1 \leq i \leq n+1$, is a component of $V-W$. Suppose $X_{i}^{\prime}(1 \leq i \leq n+1)$ with $\#\left(X_{i}^{\prime}\right) \geq 2$ exists. Let $j$ be an element of $X_{i}^{\prime}$. Put $V^{\prime}=V-\{j\}$. Note that $W \subset V^{\prime}$. We consider the simplicial complex $\Delta^{\prime}=\Delta(C(v-1, d))$ on the vertex set $V^{\prime}$. Then we have $\Delta_{W}^{\prime}=\Delta_{W}$ by Lemma 2.1 . Thus we have $\tilde{H}_{j}\left(\Delta_{W} ; k\right)=$ $\tilde{H}_{j}\left(\Delta_{W}^{\prime} ; k\right)$. By the induction hypothesis, we are done in this case.

We put $X_{0}:=Y_{1}, X_{n+1}:=Y_{2}$. Next suppose $X_{i}(0 \leq i \leq n+1)$ with \# $\left(X_{i}\right) \geq 2$ exists. Let $j$ be an element of $X_{i}$. Put $V^{\prime}=V-\{j\}$. We consider the simplicial complex $\Delta^{\prime}:=\Delta(C(v-1, d))$ on the vertex set $V^{\prime}$. Then we have $\Delta_{V-W}^{\prime}=\Delta_{V-W}$ by Lemma 2.1. By Alexander duality, we have

$$
\tilde{H}_{[d / 2]-1}\left(\Delta_{W} ; k\right) \cong \tilde{H}_{[d / 2]}\left(\Delta_{V-W} ; k\right)=\tilde{H}_{[d / 2]}\left(\Delta_{V-W}^{\prime} ; k\right) \cong \tilde{H}_{[d / 2]-1}\left(\Delta_{W}^{\prime} ; k\right) .
$$

Thus we are done in this case.

In the remaining case we may assume $\#\left(X_{i}\right)=1$ for $1 \leq i \leq n, \#\left(X_{i}^{\prime}\right)=1$ for $1 \leq i \leq n+1$ and $\#\left(Y_{i}\right) \leq 1$ for $i=1,2$. However, in this case we have the desired result by Lemmas 3.4-3.7.

Proof of Theorem 3.2. Since $k[\Delta]$ is Gorenstein (see, e.g., Corollary 5.5.6 of [BHe]), we have $\operatorname{hd}_{A} k[\Delta]=v-d$. Let

$$
0 \rightarrow F_{v-d} \rightarrow \cdots \rightarrow F_{2} \rightarrow F_{1} \rightarrow A \rightarrow k[\Delta] \rightarrow 0
$$

be a minimal free resolution of $k[\Delta]=A / I_{\Delta}$ over $A$. By Lemma 2.2, we have $\min \{\alpha \epsilon$ $\left.\mathbf{Z} ;\left(I_{\Delta}\right)_{\alpha} \neq 0\right]=[d / 2]+1$. Then $F_{1}$ has a direct summand of the form $A(-[d / 2]-1)^{b_{1}}$ with $b_{1}>0$ and $F_{i}, 1 \leq i \leq v-d-1$, may have $A(-[d / 2]-i)^{b_{i}}$ with $b_{i} \geq 0$ as a direct summand. We have $F_{v-d}=A(-v)$ and $F_{i}, 1 \leq i \leq v-d-1$, may have $A(-v+[d / 2]+(v-d-i))^{b_{v-d-i}}=A(-[d / 2]-i-1)^{b_{v-d-i}}$ as a direct summand by the self-duality of the minimal free resolution (see, e.g., p. 59 of [St]). By Proposition 1.1 of [BH2] we can easily check that other shiftings do not appear, since $k[\Delta]$ is Gorenstein. Thus we obtain the desired form of the minimal free resolution of $k[\Delta]$.

We now determine the graded Betti numbers $b_{i}, 1 \leq i \leq v-d-1$. By Hochster's formula we have

$$
b_{i}=\beta_{i_{[d / 2]+i}}=\sum_{W \subset V, \#(W)=[d / 2]+i} \operatorname{dim}_{k} \tilde{H}_{[d / 2]-1}\left(\Delta_{W} ; k\right) .
$$

Let $c_{i}(n)$ denote the number of $([d / 2]+i)$-subsets $W$ of $V$ such that $W$ has a unique decomposition $W=X_{1} \cup X_{2} \cdots \cup X_{n}$ where $X_{i}, 1 \leq i \leq n$, is a contiguous subset of $W$. Then $c_{i}(n)$ is the number of positive integer solutions of the system of the equations

$$
\left\{\begin{array}{l}
x_{1}+x_{2}+\cdots+x_{n}=\left[\frac{d}{2}\right]+i, \\
y_{1}+y_{2}+\cdots+y_{n+1}=v-\left[\frac{d}{2}\right]-i .
\end{array}\right.
$$

Thus we have

$$
c_{i}(n)=\left(\begin{array}{c}
{[d / 2]+i-1} \\
n-1
\end{array}\right)\left(\begin{array}{c}
v-[d / 2]-i-1 \\
n
\end{array}\right)
$$


By Lemma 3.8 and combinatorial identities in Appendix 3 of $[\mathrm{Br}]$ we have

$$
\begin{aligned}
b_{i} & =\sum_{n \geq 1} c_{i}(n)\left(\begin{array}{c}
n-1 \\
{[d / 2]}
\end{array}\right) \\
& =\sum_{n \geq 1}\left(\begin{array}{c}
v-[d / 2]-i-1 \\
n
\end{array}\right)\left(\begin{array}{c}
{[d / 2]+i-1} \\
n-1
\end{array}\right)\left(\begin{array}{c}
n-1 \\
{[d / 2]}
\end{array}\right) \\
& =\sum_{n \geq 1}\left(\begin{array}{c}
v-[d / 2]-i-1 \\
n
\end{array}\right)\left(\begin{array}{c}
{[d / 2]+i-1} \\
{[d / 2]}
\end{array}\right)\left(\begin{array}{c}
i-1 \\
n-[d / 2]-1
\end{array}\right) \\
& =\left(\begin{array}{c}
{[d / 2]+i-1} \\
{[d / 2]}
\end{array}\right)\left(\begin{array}{c}
v-[d / 2]-2 \\
i+[d / 2]
\end{array}\right) .
\end{aligned}
$$

\section{Unimodality of Betti Number Sequences}

In this section we show unimodality of the Betti number sequence $\left(\beta_{0}, \beta_{1}, \ldots, \beta_{v-d}\right)$ of the Stanley-Reisner ring $k[\Delta(C(v, d))]$ associated with the cyclic polytope $C(v, d)$. Since this sequence is symmetric, i.e., $\beta_{i}=\beta_{v-d-i}$ for every $0 \leq i \leq v-d$, the unimodality means $\beta_{0} \leq \beta_{1} \leq \cdots \leq \beta_{[(v-d) / 2]}$.

Lemma 4.1. Suppose $d$ is odd. With the same situation as in Theorem 3.2 we have the following:

(1) If $v-d$ is even, then

$$
b_{1}<b_{2}<\cdots<b_{(v-d) / 2}>b_{(v-d+2) / 2}>\cdots>b_{v-d-1} .
$$

(2) If $v-d$ is odd, then

$$
b_{1}<b_{2}<\cdots<b_{(v-d-1) / 2}>b_{(v-d+1) / 2}>\cdots>b_{v-d-1} .
$$

Proof. This lemma is clear from the following observation.

$$
\begin{aligned}
\frac{b_{i}}{b_{i+1}} & =\frac{i(i+1+[d / 2])}{([d / 2]+i)(v-2[d / 2]-2-i)}>1 \\
& \Leftrightarrow v-2\left[\frac{d}{2}\right]-2-i<i\left(\frac{[d / 2]+i+1}{[d / 2]+i}\right) \\
& \Leftrightarrow v-2\left[\frac{d}{2}\right]-2-i \leq i \\
& \Leftrightarrow \frac{v-2[d / 2]-2}{2} \leq i \\
& \Leftrightarrow \frac{v-d-1}{2} \leq i .
\end{aligned}
$$


Corollary 4.2. Let $\Delta$ be the boundary complex $\Delta(C(v, d))$ of the cyclic polytope $C(v, d)$. Then, the Betti number sequence $\left(\beta_{0}(k[\Delta]), \beta_{1}(k[\Delta]), \ldots, \beta_{v-d}(k[\Delta])\right)$ of the Stanley-Reisner ring $k[\Delta]$ over $A$ is unimodal.

Proof. Suppose $d$ is odd. Then $\beta_{i}=b_{i}+b_{v-d-i}$ for $1 \leq i \leq v-d-1$. Now the corollary is clear from the above lemma.

Suppose $d$ is even. By Proposition 3.1 and Theorem 3.2, we see $\beta_{i}(k[\Delta])=$ $\beta_{i}(k[\Delta(C(v+1, d+1))])$ for $0 \leq i \leq v-d$. Thus we can reduce this case to the odd-dimensional case.

\section{References}

[Ba] K. Baclawski, Cohen-Macaulay connectivity and geometric lattices, European J. Combin. 3 (1982), 293-305.

[BL] L. J. Billera and C. W. Lee, A proof of the sufficiency of McMullen's conditions for $f$-vectors of simplicial convex polytopes, J. Combin. Theory Ser. A 31 (1981), 237-255.

[Br] A. Brøndsted, An Introduction to Convex Polytopes, Springer-Verlag, New York, 1982.

[BHe] W. Bruns and J. Herzog, Cohen-Macaulay Rings, Cambridge University Press, Cambridge, 1993.

[BH1] W. Bruns and T. Hibi, Cohen-Macaulay partially ordered sets with pure resolutions, Preprint, October, 1993.

[BH2] W. Bruns and T. Hibi, Staniey-Reisner rings with pure resolutions, Comm. Algebra 23 (1995), 12011217.

[H1] T. Hibi, Algebraic Combinatorics on Convex Polytopes, Carslaw, Glebe, N.S.W., 1992.

[H2] T. Hibi, Cohen-Macaulay types of Cohen-Macaulay complexes, J. Algebra 168 (1994), 780-797.

[H3] T. Hibi, Canonical modules and Cohen-Macaulay types of partially ordered sets, Adv. in Math. 106 (1994), 118-121.

[H4] T. Hibi, Buchsbaum complexes with linear resolutions, J. Algebra, to appear.

[Ho] M. Hochster, Cohen-Macaulay rings, combinatorics, and simplicial complexes, in Ring Theory $I I$ (B. R. McDonald and R. Morris, eds.), Lecture Notes in Pure and Applied Mathematics, No. 26, Dekker, New York, 1977, pp. 171-223.

[M] J. Munkres, Topological results in combinatorics, Michigan Math. J. 31 (1984), 113-128.

[Sc] P. Schenzel, Über die freien Aufösungen extremaler Cohen-Macaulay-Ringe, J. Algebra 64 (1980), 93-101.

[St] R. P. Stanley, Combinatorics and Commutative Algebra, Birkhäuser, Boston, 1983.

[TH1] N. Terai and T. Hibi, Stanley-Reisner rings whose Betti numbers are independent of the base field, Discrete Math., to appear.

[TH2] N. Terai and T. Hibi, Alexander duality theorem and second Betti numbers of Stanley-Reisner rings, Adv. in Math., to appear.

[TH3] N. Terai and T. Hibi, Computation of Betti numbers of monomial ideals associated with stacked polytopes, Preprint, January, 1995.

Received July 21, 1994, and in revised form January 18, 1995.

Note added in proof. After submitting this article, we obtained the "stacked polytope" version of our results. See [TH3]. 